\title{
Induction of Biofilm Formation in Klebsiella pneumoniae by Acetaminophen. Quorum Quenching Compounds to Overcome the Resistance ${ }^{+}$
}

\author{
Fernando Echeverri 1,*, Elizabeth Cadavid 2, Sara Robledo ${ }^{2}$, Wiston Quiñones ${ }^{2}$ \\ 1 Grupo de Química Orgánica de Productos Naturales, Instituto de Química, Universidad de Antioquia, \\ Calle 67 No. 53-10, Medellín 050010, Colombia \\ 2 PECET, Instituto de Investigaciones Médicas, Facultad de Medicina, Universidad de Antioquia, Calle 62 \\ No. 52-59, Medellín 050010, Colombia \\ * Correspondence: fernando.echeverri@udea.edu.co; Tel.: +57-42196595 \\ + Presented at the 2nd Molecules Medicinal Chemistry Symposium (MMCS): Facing Novel Challenges in \\ Drug Discovery, Barcelona, Spain, 15-17 May 2019.
}

Published: 8 August 2019

Keywords: Klebsiella pneumoniae; resistance; biofilm; drug; acetaminophen; inhibition; furanone and cinnamic acid, derivatives

Bacterial resistance to antibiotics is a serious world health problem; consequently, there is a high recurrence of nosocomial infections. For this reason, the WHO has launched a warning to look for new molecules and mechanisms of action against bacterial pathogens. On the other hand, Quorum Sensing is a bacterial communication process involved in pathogenesis and virulence; it is mediated by several types of molecules, mainly lactones, quinolones, peptides, and boron derivatives, among others. In addition, the use of inhibitors or Quorum Quenching compounds as an alternative to overcome this type of resistance has been proposed. Thus, we consider that some medicines might also be responsible for bacteria resistance acting like inducers of Quorum Sensing. Therefore, in this work, we aimed to determine the role of some drugs in the resistance of Klebsiella pneumoniae and to establish the effects of several natural products against this pathogen bacteria.

Effectively, some drugs, especially acetaminophen, induced the biofilm formation, the synthesis of an autoinducer lactone, the colonization of urethral catheters, and increased the resistance of biofilm to gentamicin. However, several compounds were also able to counteract the effect of acetaminophen and even weaken the formed biofilm.

Funding: This research was sponsored by COLCIENCIAS (Colombia) through contract 641-2018. EC also thanks for a fellowship in the Doctoral Formation Program (567-2012). 